\title{
Impact of CYP3A5 Gene Polymorphism on Efficacy of Simvastatin
}

\author{
Genovefa Kolovou $^{1, *}$, Georgia Ragia ${ }^{2}$, Vana Kolovou ${ }^{1,3}$, Constantinos Mihas ${ }^{4}$, Niki Katsiki ${ }^{5}$, \\ Ioannis Vasiliadis ${ }^{1}$, Sophie Mavrogeni ${ }^{1}$, Vassiliki Vartela ${ }^{1}$, Anna Tavridou ${ }^{1}$ and \\ Vangelis G. Manolopoulos 2
}

${ }^{I}$ Cardiology Department, Onassis Cardiac Surgery Center Athens, Greece; ${ }^{2}$ Laboratory of Pharmacology, Medical School, Democritus University of Thrace, Alexandroupolis, Greece; ${ }^{3}$ Molecular Immunology Laboratory, Onassis Cardiac Surgery Center Athens, Greece; ${ }^{4}$ Internal Medicine Department, Kimi General Hospital, Kimi, Greece; ${ }^{5}$ Second Propedeutic Department of Internal Medicine, Medical School, Aristotle University of Thessaloniki, Hippocration Hospital, Thessaloniki, Greece

\begin{abstract}
Background: }}$ One of the promises of human genetics is individualized therapy. Therefore, we evaluated the impact of CYP3A5 gene polymorphism on the effectiveness of simvastatin (a HMG-CoA reductase inhibitor). Methods: Patients $(\mathrm{n}=191)$ with hypercholesterolemia were treated with simvastatin for at least 6 months and were genotyped for the CYP3A5 polymorphism. Results: The frequency of CYP3A5 polymorphism was $0.5 \%$ for $W T$ (wild-type), $15.6 \%$ for $H T$ (heterozygous, expressors) and $83.9 \%$ for $H M$ (homozygous, non-expressors). Differences in lipid profile before and after dose-response of simvastatin treatment were described as \% difference $\{[$ (variable after-variable before)/variable before $\left.{ }^{*} 100\right\}$. There was a trend towards the decrease of low density lipoprotein cholesterol (LDL-C) in $H T$ individuals who had a $-35.2 \%$ reduction with a dose of $20 \mathrm{mg}$ simvastatin and $H M$ individuals who had a slightly higher decrease (-37.5\%) despite the lower dose of simvastatin $(10 \mathrm{mg}, \mathrm{p}=0.07)$. Furthermore, $H T$ genotype individuals had significantly higher than expected (6-8\%) LDL-C \% difference between 20 and $40 \mathrm{mg}$ of simvastatin $(-35.2 \mathrm{vs}-49.2 \%, \mathrm{p}=0.037)$. In individuals with $H M$ genotype a significant LDL-C \% difference was found between 10 and $40 \mathrm{mg}$ of simvastatin (-37.5 vs $48.4 \%, \mathrm{p}=0.023)$. Conclusion: The individuals with $H M$ polymorphism display a trend towards higher LDL-C reductions compared with $H T$ polymorphism. Within the same genotype, differences between doses were also observed. These findings need to be confirmed in larger studies.
\end{abstract}

Keywords: Simvastatin, CYP3A5 gene polymorphism, low density lipoprotein cholesterol.

\section{INTRODUCTION}

HMG-CoA (3-hydroxy-3-methylglutaryl coenzyme A) reductase inhibitors (statins) are the first-line drugs for hypercholesterolemia treatment. However, there is considerable variation in the reduction of plasma low density lipoprotein cholesterol (LDL-C) concentration in response to statin treatment (from - 25 to - 60\%) [1]. Even more confusing is the efficacy of statins on triglycerides (TGs) reduction, varying from - 10 to $-50 \%$, and high density lipoprotein cholesterol (HDL-C) varying from -1 to $+15 \%[2,3]$. Environmental and genetic factors may contribute to this variability.

Simvastatin is one of the oldest lipophilic statins, which enters and exits hepatocytes through passive diffusion $[1,2]$. Simvastatin is extensively metabolized by two enzymes of the cytochrome P450 superfamily that catalyze the oxidation of organic substances, the CYP3A4 and CYP3A5 which are encoded by the CYP3A4 and CYP $3 A 5$ genes, respectively [4]. The most common reaction catalyzed by cytochromes $\mathrm{P} 450$ is a monooxygenase reaction. The $\mathrm{P} 450$ proteins are localized in the cell endoplasmic reticulum and their

*Address correspondence to this author at the Onassis Cardiac Surgery Center, 356 Sygrou Ave 17674 Athens, Greece;

Tel: +30 210 9493520; Fax: +30 2109493336

E-mail: genovefa@kolovou.com expression is induced by glucocorticoids and various pharmacological agents $[5,6]$. The $C Y P 3 A 5$ gene is part of a cluster of cytochrome $P 450$ genes on chromosome $7 \mathrm{q} 21.1$. This cluster includes a pseudogene, CYP3A5P1 and CYP3A5. There are substantial CYP3A expression differences between individuals that contribute to the variation in bioavailability and clearance of CYP3A substrates. A polymorphism in $C Y P 3 A 5$, the CYP3A5*3 allele, is the major factor that modulates $\mathrm{P} 450$ proteins expression [7]. Also, CYP $3 A 5$ plays an important role in the appearance of adverse effects.

Statins have been reported to increase CYP3A expression in vitro as ligands to nuclear receptors (pregnane $\mathrm{X}$ receptor and constitutive androsterone receptor) that form heterodimers with retinoid $X$ receptors and bind to responsive elements in the CYP3A4 and CYP3A5 promoter regions [8]. Individuals with at least one $C Y P 3 A 5 * 1$ allele express large amounts of CYP3A5. Thus, the CYP3A5 may be one of the important genetic contributors to inter-individual and interracial differences in CYP3A-dependent drug responses and clearance. Despite the significant research that has been performed to find out the function of P450 genes, few studies have evaluated the practical value of various factors involved in controlling P450 expression and variation among individuals [9]. 
The aims of this study were:

1. To investigate the impact of $C Y P 3 A 5$ gene polymorphism on the effectiveness of simvastatin in patients with primary hypercholesterolemia.

2. To explore the influence of genetic variations of $C Y P 3 A 5$ gene on the response to simvastatin treatment by examining genotype frequencies in hyper-responders and hyporesponders.

\section{METHODS}

\section{Subjects}

The number of participants needed to achieve $90 \%$ statistical power at a significance level of $5 \%$ and to evaluate differences higher than 5\% in total cholesterol (TC) values after treatment was 190 . Therefore, we consecutively selected and genotyped 191 Greek unrelated subjects [125 men and 66 women; median age \pm interquartile range $\left(75^{\text {th }}-25^{\text {th }}\right.$ percentile, IQR) $=60.0$ years \pm 17.0$)]$ with primary hypercholesterolemia who attended the Lipid Clinic of the Cardiology Department at Onassis Cardiac Surgery Center. They gave their consent before entering the study. Additional inclusion criteria were a stable medication, which was unlikely to interfere with lipid profile (patients with coronary heart disease were on cardioselective $\beta$-blockers and aspirin, whereas patients with hypertension were on angiotensin converting enzyme inhibitors) and routine lifestyle for at least 4 weeks prior to study screening. Subjects with a history of renal or thyroid disease and uncontrolled diabetes mellitus were excluded from the study. Subjects were assigned to simvastatin treatment for at least 6 months $[9 \pm 2$ (SD), Standard Deviation]. The dose of simvastatin (10-40 $\mathrm{mg} /$ day) was adjusted according to the National Cholesterol Education Program Adult Treatment Panel III (NCEP ATP III) treatment goal for LDL-C based on risk category (LDL-C $<130,<100$ or $<$ $70 \mathrm{mg} / \mathrm{dl} ;<3.4,<2.6$ or $<1.8 \mathrm{mmol} / 1)$ [10]. The lipids were monitored every 3 months in order to titrate simvastatin dose. All subjects were taking simvastatin as the only lipidlowering drug. Furthermore, according to LDL-C changes, patients were divided to Hyper-responders and Hyporesponders.

Our Institutional Review Board approved the study.

\section{Determination of Blood Lipids and Glucose}

Total cholesterol (TC), TGs and HDL-C levels were measured using enzymatic colorimetric methods, on a Roche Integra Biochemical analyzer, with commercially available kits (Roche Diagnostics Gmbh, Hannheim, Germany). The serum LDL-C levels were calculated using the Friedewald formula in subjects with TG levels $<400 \mathrm{mg} / \mathrm{dl}(4.5 \mathrm{mmol} / \mathrm{l})$.

\section{Genotyping of CYP3A5*3 Polymorphism}

Five millilitre blood samples were collected by direct venipuncture from each patient and were drawn in a vacutainer tube containing ethylene diaminetetraacetic acid (EDTA). DNA was extracted by using QIAGENFlexiGeneDNA kit.

Polymerase chain reaction (PCR) was used to amplify the sequence of interest as described previously [9]. The following pair of primers was used for amplification: 5'-
CATCAGTTAGTAGACAGATGA-3' (forward) and 5'GGTCCAAACAGGGAAGAAAA-3' (reverse). The underlined nucleotide is a mismatch with CYP3A5 sequence, creating an additional restriction site in the PCR product. The reaction mix was composed by: $5 \mu 110 x$ buffer, $1.5 \mu 1$ $\mathrm{MgCl}_{2} 1 \mathrm{mM}, 0.4 \mu \mathrm{ldNTPs} 25 \mathrm{mM}, 0.5 \mu \mathrm{l}$ forward primer $115 \mathrm{pmol} / \mu \mathrm{l}, 0.5 \mu \mathrm{l}$ reverse primer $134 \mathrm{pmol} / \mu \mathrm{l}, 0.5 \mu \mathrm{Taq}$ polymerase (5U-HyTest), $3 \mu \mathrm{l}$ DNA (final volume: $50 \mu \mathrm{l}$ ). PCR started with initial denaturation at $94^{\circ} \mathrm{C} / 5 \mathrm{~min}$ and followed by 40 cycles under the following conditions: denaturation at $94^{\circ} \mathrm{C} / 1 \mathrm{~min}$, annealing at $55^{\circ} \mathrm{C} / 1 \mathrm{~min}$ and extension at $72{ }^{\circ} \mathrm{C} / 1 \mathrm{~min}$. The final extension was carried out at 72 ${ }^{\circ} \mathrm{C} / 10 \mathrm{~min}$. All PCR amplifications were carried in the PCRengine apparatus PTC-200 of MJ Research (Watertown, Mass., USA).

The PCR product (293 bps) of $10 \mu \mathrm{l}$ was digested in a 20 $\mu l$ reaction volume with $5 \mathrm{U}$ of SspI restriction enzyme (Takara Bio Inc., Shiga, Japan) at $37{ }^{\circ} \mathrm{C}$ for $2.5 \mathrm{~h}$. The digested products were resolved on $2.5 \% \mathrm{w} / \mathrm{v}$ agarose gel electrophoresis, visualized by staining with ethidium bromide and identified with a 25 bps molecular weight ladder (Invitrogen). The fragments obtained for CYP3A5*1 allele were 148, 125 and $20 \mathrm{bps}$, while for $C Y P 3 A 5 * 3$ allele were 168 and $125 \mathrm{bps}$. An internal positive to $C Y P 3 A 5 * 1$ allele (rare allele) control was used in each PCR-RFLP run.

\section{Statistical Analysis}

All continuous variables are presented as median \pm interquartile range $\left(75^{\text {th }}-25^{\text {th }}\right.$ percentile, IQR) since they deviated from normal distribution. All categorical variables are presented as absolute and relative (percentage) frequencies. Mann-Whitney $U$ and Wilcoxon rank-sum statistics were used in order to evaluate the differences in continuous variables between different groups and between baseline and after treatment, respectively. The differences in TC, TGs, HDL-C and LDL-C before and after simvastatin treatment were also described as \% difference, based on the following rule: $\%$ difference $=[($ variable after-variable before $) /$ variable before $]^{*} 100$. All tests were two-sided at a significance level of $\mathrm{p}<0.05$. Data were analyzed using STATA ${ }^{\mathrm{TM}}$ statistical software (Version 9.0, Stata Corporation, College Station, TX 77845, USA).

\section{RESULTS}

The TC, LDL-C and TG levels were significantly decreased with simvastatin treatment $(-33.1,-42.9$ and $20.2 \%$, respectively, $\mathrm{p}<0.001)$, while HDL-C concentration increased $(+1.6 \%, \mathrm{p}=0.01)$, (Table 1$)$.

\section{Simvastatin Dose}

The majority of the individuals (51.4\%) were on $20 \mathrm{mg}$ daily, whereas $22.9 \%$ were on $10 \mathrm{mg}$ and $25.7 \%$ on $40 \mathrm{mg}$ daily.

\section{Genotype Frequencies}

The genotype frequency of the $C Y P 3 A 5$ polymorphism was for homozygous ( $H M$, non-expressors) $83.9 \%$, for heterozygous (HT, expressors) $15.6 \%$ and for wild-type $(W T)$ $0.5 \%$. The $W T$ genotype was excluded from analysis due to small number $(\mathrm{n}=1)$. 
Table 1. Patients characteristics and lipid values before and after simvastatin treatment.

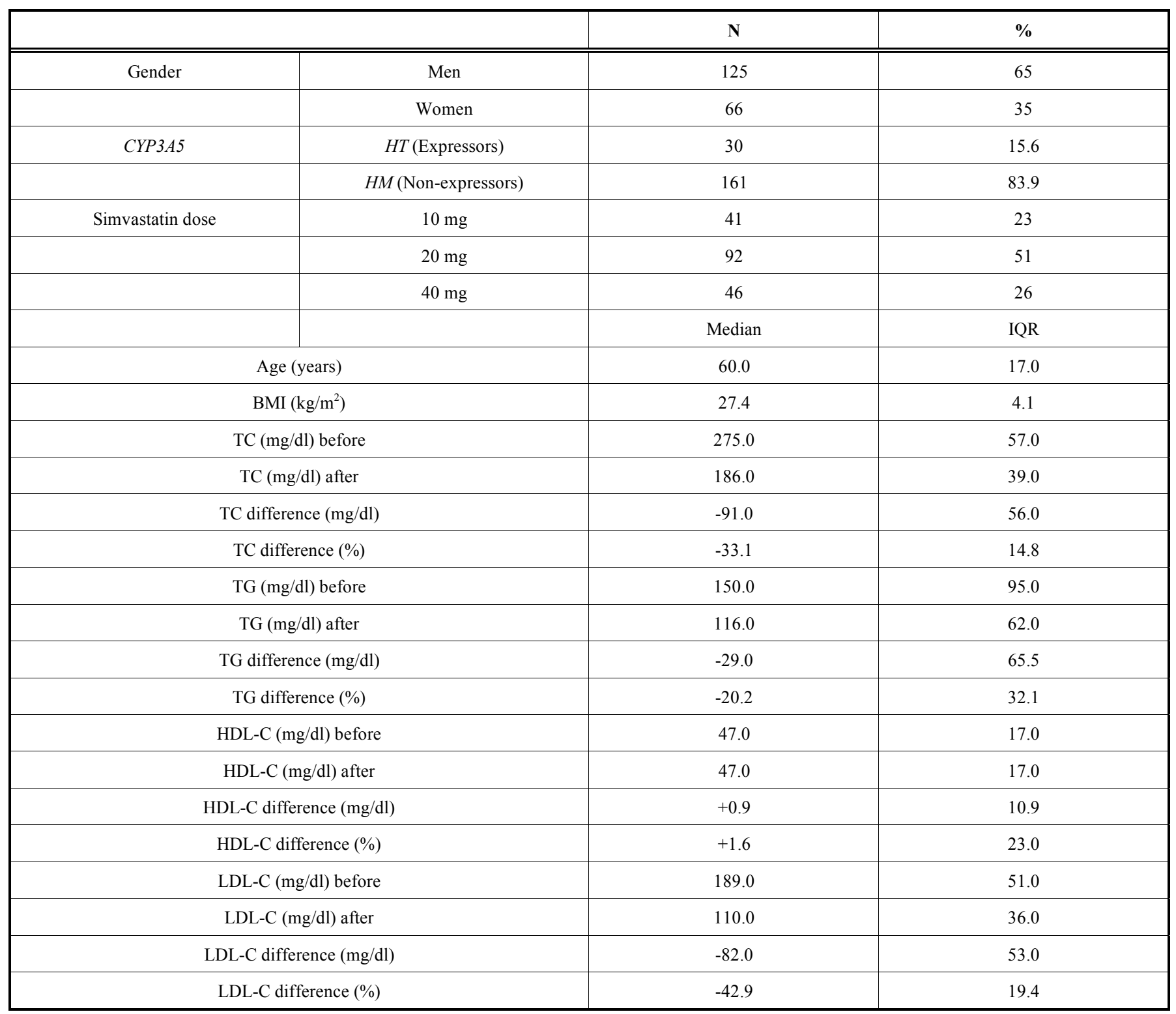

BMI $=$ Body Mass Index; TC $=$ Total Cholesterol; TG $=$ Triglycerides; HDL-C $=$ High Density Lipoprotein Cholesterol; LDL-C $=$ Low Density Lipoprotein Cholesterol. WT genotype was excluded from the analysis due to the small number $(\mathrm{n}=1)$.

\section{Patient Characteristics}

Baseline characteristics of the total study patients are shown in (Table 1).

TC, TGs, HDL-C and LDL-C concentrations as well as $\%$ differences before and after simvastatin treatment based on genotype are shown in (Table $\mathbf{2 A}$ and Table $\mathbf{2 B}$ ).

The \% differences within $H M$ and $H T$ genotypes are shown in (Table 3 ).

\section{Dose-response According to CYP3A5 Genotypes}

The individuals with $H M$ polymorphism displayed a trend towards higher LDL-C reductions compared with $H T$ polymorphism $(\mathrm{p}=0.07)$. The $H T$ genotype individuals (expressors) had a - 35.2\% LDL-C decrease with $20 \mathrm{mg}$ simvastatin, whereas $H M$ individuals (non-expressors) displayed a relatively higher decrease (- 37.5\%) with the half dose of simvastatin (i.e. $10 \mathrm{mg}$ ).

The $H T$ genotype individuals had significantly higher than expected (as doubling the statin dose is adding further lowering of the LDL-C only by $6-8 \%$, additionally to the first dose [11]) LDL-C \% difference between 20 and $40 \mathrm{mg}$ of simvastatin (- $35.2 \mathrm{vs}-49.2 \% ; \mathrm{p}=0.037)$. In individuals with $H M$ genotype the significant LDL-C \% difference was between 10 and $40 \mathrm{mg}(-37.5 \mathrm{vs}-48.4 \%$; $\mathrm{p}=0.023)$.

\section{Hyper-responders and Hypo-responders}

Hyper-responders were considered those in the upper percentile (Min-25th percentile; from - 80.3 to - 51.5\%) and as Hypo-responders were considered those in the lower percentile (75th percentile-maximum; from - 32.1 to $93.5 \%$ ). No $\%$ differences were found according to dose of 
Table 2A. Descriptive statistics by genotype.

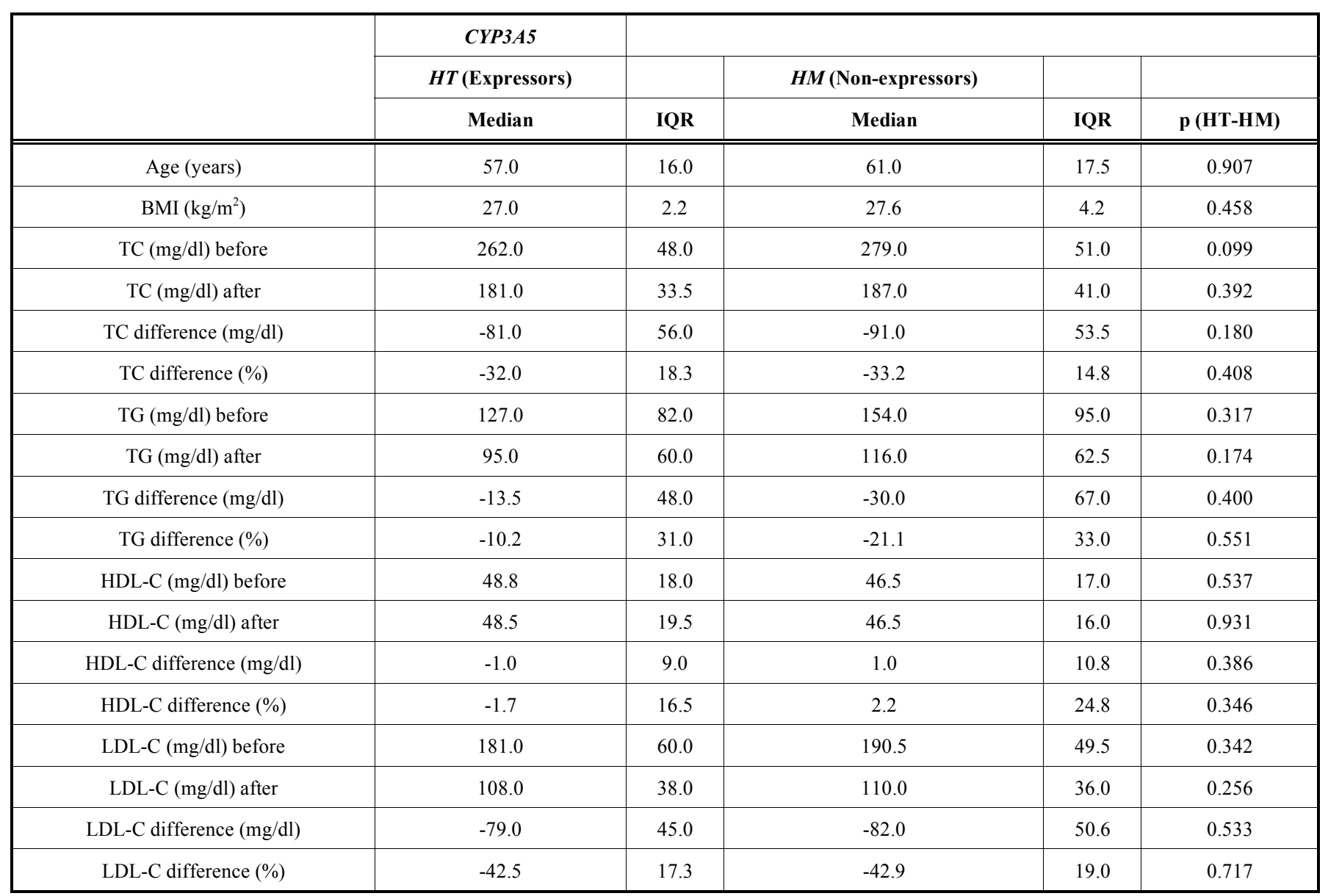

BMI = Body Mass Index; TC = Total Cholesterol; TG = Triglycerides; HDL-C = High Density Lipoprotein Cholesterol; LDL-C = Low Density Lipoprotein Cholesterol. WT genotype was excluded from the analysis due to the small number $(\mathrm{n}=1)$.

Table 2B. Significance of differences [baseline (b), afterwards (a)] for each genotype.

\begin{tabular}{|c|c|c|}
\hline \multirow{2}{*}{} & \multicolumn{2}{|c|}{ CYP3A5 } \\
\cline { 2 - 3 } & $\mathbf{p}$ & HM (Non-expressors) \\
\cline { 2 - 3 } & $<$ & $\mathbf{p}$ \\
\hline \hline TC b-a & $<0.001$ & $<0.001$ \\
\hline TG b-a & 0.003 & $<0.001$ \\
\hline HDL-C b-a & 0.629 & 0.202 \\
\hline LDL-C b-a & $<0.001$ & $<0.001$ \\
\hline
\end{tabular}

TC = Total Cholesterol; TG = Triglycerides; HDL-C = High Density Lipoprotein Cholesterol; LDL-C = Low Density Lipoprotein Cholesterol.

WT genotype was excluded from the analysis due to the small number $(\mathrm{n}=1)$.

simvastatin and genotypes in Hyper-responders and Hyporesponders (Table 4$)$.

All patients were on hypolipidemic diet before entering the study; therefore the lipid profile changes after simvastatin treatment were not influenced by diet. Furthermore, the BMI was constant during the whole study.

\section{DISCUSSION}

We evaluated the impact of CYP3A5 gene polymorphism on the effectiveness of simvastatin treatment in subjects with primary hypercholesterolemia. The genotype frequencies for the CYP3A5 gene polymorphism in our study cohort were similar to those reported in other Caucasian $[8,12,13]$ and dyslipidemic populations [14]. Concerning the impact of CYP gene polymorphism on effectiveness of simvastatin treatment, the results are still not clear $[15,16]$. Li et al. [15] compared the lipid lowering efficacy of simvastatin (202 patients) and atorvastatin (177 patients) according to $C Y P 3 A P 1 * 3$ (non-expressors) variant allele in Chinese hyperlipidemic patients. They reported that in women treated 
Table 3. CYP3A5 genotype and simvastatin dose.

\begin{tabular}{|c|c|c|c|c|c|}
\hline \multirow{2}{*}{} & & & \multicolumn{3}{|c|}{ Simvastatin Dose } \\
\cline { 3 - 6 } & & & $\mathbf{1 0} \mathbf{~ m g}$ & $\mathbf{2 0} \mathbf{~ m g}$ & $\mathbf{4 0} \mathbf{~ m g}$ \\
\hline \hline CYP3A5 & $H T$ (Expressors) & $\mathrm{N}$ & 9 & 10 & 9 \\
\hline & & $\%$ & $22 \%$ & $20 \%$ & 82 \\
\hline & $H M$ (Non-expressors) & $\mathrm{N}$ & 32 & $89 \%$ & $80 \%$ \\
\hline
\end{tabular}

$\mathrm{p}=0.186$ between $H T$ and $H M$ genotypes according to simvastatin dose. $\%=$ frequency of CYP3A5 genotypes within simvastatin dose. WT genotype was excluded from the analysis due to the small number $(\mathrm{n}=1)$.

Table 4. Low density lipoprotein cholesterol response by $C Y P 3 A 5$ genotypes.

\begin{tabular}{|c|c|c|c|}
\hline & CYP3A5 & CYP3A5 & HT (Expressors) \\
\hline \hline & $\boldsymbol{H M}$ (Non-expressors) & $\%$ & $\mathrm{~N}$ \\
\hline Hyper-responders & $\mathrm{N}$ & $51 \%$ & 6 \\
\hline Hypo-responders & 31 & $49 \%$ & $43 \%$ \\
\hline
\end{tabular}

$\mathrm{p}=0.591$, between frequency of CYP3A5 genotypes in Hyper-responders and Hypo-responders. Hyper-responders: Difference in LDL-C (Low Density Lipoprotein Cholesterol) values (\%) after treatment: from -80.3 to $-51.5 \%$ (minimum value and $25^{\text {th }}$ percentile, respectively).

Hypo-responders: Difference in LDL-C values (\%) after treatment: from - 32.1 to $93.5 \%\left(75^{\text {th }}\right.$ percentile and maximum value, respectively).

with simvastatin, the $\%$ reduction of LDL-C level was greater in the $C Y P 3 A P 1 * 3 / * 3$ compared with $C Y P 3 A P 1 * 1$ genotypes. Kivisto et al. [16] also evaluated whether the expression of $C Y P 3 A 5$ is associated with an impaired lipidlowering response to statins in 69 Caucasian patients. They found that lovastatin, simvastatin and atorvastatin were significantly less effective in $C Y P 3 A 5$ expressors than in nonexpressors. The mean serum TC concentration at 1 year was $23 \%$ higher and the mean serum LDL-C concentration was $24 \%$ higher in subjects possessing the $C Y P 3 A 5$ expressors than in non-expressors. The mean $\%$ reduction in serum TC from baseline was significantly smaller in CYP3A5 expressors than in non-expressors.

Similarly to Kivisto et al. [16], we also found a trend towards lower \% reduction in LDL-C of the $H T$ (expressors) compared with $H M$ (non-expressors). The $H T$ individuals had a $-35.2 \%$ decrease with a dose of $20 \mathrm{mg}$ simvastatin while the $H M$ individuals had slightly higher decrease $(-37.5 \%)$ in spite the lower dose of simvastatin $(10 \mathrm{mg})$.

In contrast, Shin et al. [17] evaluated the efficacy of atorvastatin and found that the CYP3A5 genotype has minimal effects on the pharmacokinetic parameters of atorvastatin and its interaction with clarithromycin. However, Willrich et al. [13] reported that $C Y P 3 A 5 * 3 A$ allele was associated with reduced cholesterol-lowering response to atorvastatin in non-African individuals. Moreover, Kim et al. [18] investigated the effect of $C Y P 3 A 5 * 3$ genotype on the pharmacokinetics of simvastatin in 22 men; they found that the area under the plasma concentration-time curve for simvastatin in the $C Y P 3 A 5 * 1 / * 1$ carriers was significantly lower than in $C Y P 3 A 5^{*} 3 / * 3$. Wang et al. [19] studied the CYP3A4 polymorphism ( $r s 35599367, C>T$ ) in 235 patients taking atorvastatin, simvastatin or lovastatin and found that $T$ carri- ers required significantly lower statin doses than non-T carriers.

Noteworthy to mention that, in our study, we found that the \% difference in LDL-C within the same genotype according to dose of simvastatin was higher than expected. Many studies [11] have documented that doubling the dose of statin further decreases LDL-C levels by $6-8 \%$. In our study we found a further $14 \%$ difference in LDL-C after doubling simvastatin dose from 20 to $40 \mathrm{mg}$.

A limitation of this study is the relatively small number of subjects. On the other hand, our study has some strengths. We performed a second PCR to avoid a misclassification of heterozygotes as homozygotes. In addition, we studied a reasonably homogenous group of individuals according to geographical and ethnic origin (the majority of patients were from Athens). Ethnic differences in gene distribution have been reported [20,21].

Generally, this type of studies has numerous limitations. Among them are gender differences (in our study the influence of gender on simvastatin treatment was not evaluated due to the relatively small study cohort), the role of absorption of cholesterol, diet, BMI, adherence to treatment, subgrouping of a small population to different doses of simvastatin, interference with other drugs or food supplements and different pathogenesis of dyslipidaemia (including combinations of primary and secondary causes). However, many of these factors should be balanced out within the groups we studied. Assessing some of these factors would also require costly and complex techniques. It follows that some of these measurements are likely to be missing from several studies.

However, polymorphisms may affect the pharmacokinetic and pharmacodynamic profiles of statins, thus creating 
a potential application in the personalized prescribing of statin therapy. Genotype evaluation may help to identify individuals with better response to treatment. It follows that the prospective identification of subjects who have a weak or enhanced response to statins may improve the risk-to-benefit ratio of statin therapy. It is still cheaper to try different statins and to increase the dose of a statin or proceed to combination therapy than to perform expensive genotyping. However, we need to understand these interrelationships because they may help to design more effective drugs. Furthermore, if we detect substantial ethnic variations then we could have different statins as first choice. Noteworthy to mention is that there is a lot more to statin action than just $C Y P 3 A 5$. For example, upregulation of cholesterol absorption and cellular transport after giving a statin involved genes such as ABCA1, ABCB1, ABCG2, SLCO1B1 [22], new players such as PCSK-9 response to a statin [23] and other factors.

\section{CONCLUSIONS}

In the present study, simvastatin was less effective in CYP3A5 expressors compared with non-expressors. The individuals with $H M$ displayed a trend towards higher LDL-C decrease with the half dose of simvastatin compared with $H T$ individuals. Within the same genotype, differences between doses were also found. Further large studies are required to clarify the contribution genes to statin-induced lipid lowering effects.

\section{CONFLICT OF INTEREST}

The author(s) confirm that this article content has no conflicts of interest.

\section{ACKNOWLEDGEMENT}

Declared none.

\section{LIST OF ABBREVIATIONS}

$$
\begin{aligned}
& \text { LDL-C }=\text { Low density lipoprotein cholesterol } \\
& \text { HDL-C }=\text { High density lipoprotein cholesterol } \\
& \text { TG }=\text { Triglyceride } \\
& \text { TC }=\text { Total cholesterol } \\
& \text { PCR }=\text { Polymerase chain reaction }
\end{aligned}
$$

\section{REFERENCES}

[1] Kolovou GD, Katerina A, Ioannis V, Cokkinos DV. Simvastatin: two decades in a circle. Cardiovasc Ther 2008; 26: 66-78.

[2] Kolovou GD, Anagnostopoulou KK, Kostakou PM, Mikhailidis DP. Cholesterol ester transfer protein (CETP), postprandial lipemia and hypolipidemic drugs. Curr Med Chem 2009; 16: 4345-60.

[3] Anagnostopoulou KK, Kolovou GD, Kostakou PM, et al. Pharmacogenetic study of cholesteryl ester transfer protein gene and simvastatin treatment in hypercholesterolaemic subjects. Expert Opin Pharmacother 2007; 8: 2459-63.

[4] Prueksaritanont T, Ma B, Yu N. The human hepatic metabolism of simvastatin hydroxy acid is mediated primarily by CYP3A, and not CYP2D6. Br J Clin Pharmacol 2003; 56: 120-4.
[5] Dvorak Z, Pavek P. Regulation of drug-metabolizing cytochrome P450 enzymes by glucocorticoids. Drug Metab Rev 2010; 42: 62135.

[6] Hukkanen J. Induction of cytochrome P450 enzymes: a view on human in vivo findings. Expert Rev Clin Pharmacol 2012; 5: 56985

[7] Kuehl P, Zhang J, Lin Y, et al. Sequence diversity in CYP3A promoters and characterization of the genetic basis of polymorphic CYP3A5 expression. Nat Genet 2001; 27: 383-91.

[8] Willrich MA, Hirata MH, Hirata RD. Statin regulation of CYP3A4 and CYP3A5 expression. Pharmacogenomics 2009; 10: 1017-24

[9] Arvanitidis K, Ragia G, Iordanidou M, et al. Genetic polymorphisms of drug-metabolizing enzymes CYP2D6, CYP2C9, CYP2C19 and CYP3A5 in the Greek population. Fundam Clin Pharmacol 2007; 21: 419-26.

[10] Grundy SM, Cleeman JI, Merz CN, et al; National Heart, Lung, and Blood Institute; American College of Cardiology Foundation; American Heart Association. Implications of recent clinical trials for the National Cholesterol Education Program Adult Treatment Panel III guidelines. Circulation 2004; 110: 227-39.

[11] Mikhailidis DP, Lawson RW, McCormick AL, et al. Comparative efficacy of the addition of ezetimibe to statin vs statin titration in patients with hypercholesterolaemia: systematic review and metaanalysis. Curr Med Res Opin 2011; 27: 1191-210.

[12] Kuehl P, Zhang J, Lin Y, et al.Frequency of common CYP3A5 gene variants in healthy Polish newborn infants. Pharmacol Rep 2009; 61: 947-51.

[13] Willrich MA, Hirata MH, Genvigir FD, et al. CYP3A53A allele is associated with reduced lowering-lipid response to atorvastatin in individuals with hypercholesterolemia. Clin Chim Acta 2008; 398: 15-20.

[14] Rosales A, Alvear M, Cuevas A, Saavedra N, Zambrano T, Salazar LA. Identification of pharmacogenetic predictors of lipid-lowering response to atorvastatin in Chilean subjects with hypercholesterolemia. Clin Chim Acta 2012; 413: 495-501.

[15] Li YP, Zhang LR, Jia M, Hu XJ. CYP3AP1*3 allele is associated with lipid-lowering efficacy of simvastatin and atorvastatin in Chinese women. J Clin Pharmacol 2011; 51: 181-8.

[16] Kivistö KT, Niemi M, Schaeffeler E, et al. Lipid-lowering response to statins is affected by CYP3A5 polymorphism. Pharmacogenetics 2004; 14: 523-5.

[17] Shin J, Pauly DF, Pacanowski MA, Langaee T, Frye RF, Johnson JA. Effect of cytochrome P450 3A5 genotype on atorvastatin pharmacokinetics and its interaction with clarithromycin. Pharmacotherapy 2011;31: 942-50.

[18] Kim KA, Park PW, Lee OJ, Kang DK, Park JY. Effect of polymorphic CYP3A5 genotype on the single-dose simvastatin pharmacokinetics in healthy subjects. J Clin Pharmacol 2007; 47: 87-93.

[19] Wang D, Guo Y, Wrighton SA, Cooke GE, Sadee W. Intronic polymorphism in CYP3A4 affects hepatic expression and response to statin drugs. Pharmacogenomics J 2011; 11: 274-86.

[20] McGraw J, Waller D. Cytochrome P450 variations in different ethnic populations. Expert Opin Drug Metab Toxicol 2012; 8: 37182.

[21] Kolovou GD, Anagnostopoulou KK, Cokkinos DV. Apolipoprotein $\mathrm{E}$ gene polymorphism and myocardial infarction. Int $\mathrm{J}$ Cardiol 2009; 133: 264-5.

[22] Zhou Q, Ruan ZR, Jiang B, Yuan H, Zeng S. Simvastatin pharmacokinetics in healthy Chinese subjects and its relations with CYP2C9, CYP3A5, ABCB1, ABCG2 and SLCO1B1 polymorphisms. Pharmazie 2013; 68: 124-8.

[23] Gelissen IC, McLachlan AJ. The pharmacogenomics of statins. Pharmacol Res 2013 Dec 21. doi: 10.1016/j.phrs.2013.12.002. [Epub ahead of print] 